\title{
Differential Scanning Calorimetric Analysis of Virgin Coconut Oil, Palm Olein, and their Adulterated Blends
}

\author{
J.M.N. Marikkar ${ }^{1 *}$, Nisa Kamil², and A.R. Raihana ${ }^{2}$
}

\begin{abstract}
Virgin coconut oil (VCO) is a premium product with a high market value. Its authenticity and quality assurance are important to safeguard consumers from fraudulent practices. The aim of this study was to investigate the effect of adulteration by palm olein (PO) on differential scanning calorimetric (DSC) heating and cooling profiles of VCO. Pure samples of VCO, PO and their adulterated blends ( 5 to $30 \%, \mathrm{w} / \mathrm{w}$ ) were subjected to thermal analysis by DSC according to a specified temperature program. DSC thermal analysis system software and SAS statistical system were used subsequently to analyze thermal data. Both cooling and heating curves of VCO were found to be vivid for fingerprint comparison of qualitative identification at $5 \%$ level of adulteration.
\end{abstract}

Key words: Authentication, adulteration, DSC, virgin coconut oil, thermal analysis

\section{Introduction}

Virgin coconut oil (VCO) is a premium product extracted from fully matured coconuts using mechanical oil expellers operating under low-temperature condition (Bawalan and Chapman, 2006). Since the oil is extracted hygienically under mild temperature conditions, it looks colorless, limpid, and free from cloudiness coming from any dirt. According to previous reports, many VCO samples were found to contain very low amounts of moisture $(<0.1)$ and free fatty acid $(<0.1)$. Other than these, the levels of hydro peroxides present in them were negligibly small to be detected (Jayasundera, et al 2003). The growing demand for VCO is mainly due to its health attributes, which arise from its triacylglycerols composition that contain considerable amounts of medium-chain fatty acids (Nevin and Rajamohan, 2004). Medium-chain triglycerides (MCT) are generally considered to be healthy since they are biologically inert source of energy that the human body finds reasonably easy to metabolize. Owing to this reason, medium-chain triglycerides have applications in treatments of a variety of malabsorption ailments (Nagao and Yanagita, 2010). Apart from the benefits on human nutrition, $\mathrm{VCO}$ is also known for its potential applications in hair oils, baby oils and massage oils used in aroma therapies. All these have caused VCO to catch up unmatched demand in the market place; this makes the oil to be vulnerable for adulteration with less expensive oils such palm olein. Palm olein, the liquid fraction of palm, is relatively cheaper to purchase due to its low cost of production. This type of economic fraud will alter the chemical composition of VCO affecting its nutritional and therapeutic values.

\footnotetext{
${ }^{1}$ Food Chemistry Laboratory, National Institute of Fundamental Studies, Hanthana Road, Kandy, Sri Lanka.

${ }^{2}$ Department of Biochemistry, Universiti Putra Malaysia, 43400 UPM Serdang, Selangor D.E., Malaysia.

*Corresponding author: nazrim.ma@nifs.ac.lk
} 
Authentication of VCO against adulteration practices has been a challenging task for many researchers in the past. For instance, detection of VCO adulteration by palm kernel oil remained as a challenge due to their close similarities in fatty acid and TAG compositions. Despite this, various analytical approaches adopted by researchers helped to provide solutions to adulteration issues ( $\mathrm{Xu}$ et al., 2011; Marina et al., 2009; Manaf et al., 2007). Differential scanning calorimetry has been one of the several instrumental techniques used by researchers for authentication of various oils and fats. As DSC is an analytical tool that can provide well-defined curves for unadulterated neat oils, it helped to give fingerprints for authentication as well as quality assurance purposes. In a pioneering effort, Dyzel and Baish (1992) demonstrated the use of DSC in the identification of various edible vegetable oils. This study triggered the curiosity of several researchers to investigate the use DSC to detect adulterations in several cases (Marikkar, 2015; Marina et al., 2009). In majority of the studies, DSC provided both cooling and heating curves that gave plenty of information about thermal transitions and associated thermal parameters to compare an authentic VCO sample with adulterated ones. Most of the time, deviations in the DSC heating and cooling profiles of suspected samples enabled the researchers to detect adulterations. Nevertheless, there has been a knowledge gap in the literature about DSC thermodynamic parameters that show highsensitivity to low-levels of adulteration by palm olein. This can be determined through the use of statistical correlation between different DSC parameters and varying levels of adulterations in VCO.

\section{Materials and Methods}

Materials: Six samples of VCO produced from 10 to 11 month old nuts were obtained from Malaysian Agricultural Research and Development Institute, Selengor, Malaysia. Six samples of PO were purchased from Lam Soon Edible Oils Sdn. Bhd, Selangor, Malaysia. All chemicals used in this study were of analytical grade unless otherwise specified (SigmaAldrich). For quantitative analysis, samples of virgin coconut oils with varying levels of adulterant oil were prepared as given below.

Blend preparation: $\mathrm{VCO}$ and adulterant (PO) were mixed together in differing ratios to form a set of samples. A total of six samples were prepared: $\left(\mathrm{A}_{1}\right)$ 95:5, $\left(\mathrm{A}_{2}\right)$ 90:10, $\left(\mathrm{A}_{3}\right)$ 85:15, $\left(\mathrm{A}_{4}\right)$ 80:20, ( $\left.\mathrm{A}_{5}\right) \quad 75: 25, \quad\left(\mathrm{~A}_{6}\right) \quad 70: 30 \quad(\mathrm{w} / \mathrm{w})$ and identified by the mass ratio of $\mathrm{VCO}$ to PO.

DSC thermal analysis: DSC analysis was performed by using Mettler Toledo DSC, Model 823 (Mettler-Toledo GmbH, Zurich, Switzerland) equipped with thermal analysis STAR $^{\mathrm{e}}$ system. Nitrogen $(99.9 \%$ purity) was used as the purge gas rate at a rate of $20 \mathrm{~mL} / \mathrm{min}$. Approximately 6-8mg of oil sample was weighed and placed in a standard DSC aluminium pan and then hermetically sealed. An empty covered DSC aluminium pan was used as a reference. In order to obtain cooling and melting profile the following temperature program was set: $70^{\circ} \mathrm{C}$ isotherm for $5 \mathrm{~min}$, cooled at $5^{\circ} \mathrm{C} / \mathrm{min}$ to $-70^{\circ} \mathrm{C}$ and held for $5 \mathrm{~min}$. The same rate was used to heat the sample from $70^{\circ} \mathrm{C}$ to $70^{\circ} \mathrm{C}$. The thermal data was analyzed using DSC software library program (Marikkar et al., 2013).

Statistical analysis: The relationships between each DSC parameter and adulteration level were determined by Pearson's correlation analysis. Statistical significance was declared at 0.05 probability level. The DSC parameters and level of adulteration were analyzed using a stepwise procedure in SAS to develop prediction models for quantification (SAS 1998). The significance level of an independent variable to enter and stay in the calibration mode was set to 0.05 during execution of the stepwise variable selection in SAS procedure "REG".

\section{Results and Discussions}

DSC analysis of lipids is generally performed under two different conditions, namely cooling process and heating process, which a give cooling curve and a melting curve, respectively. While cooling process provides details of the thermal events associated with crystallization, heating process provides information about the thermal events associated 
with melting. According to previous studies, DSC curves of most edible oils and fats are strongly influenced by their fatty acid and TAG compositions (Marikkar, 2015; Dyzel and Baish, 1992). Because of this reason, changes in lipid composition due to adulteration might cause changes in both cooling and heating curves. This has provided a scientific basis for detection of adulterations in edible oils, butter, cheese, etc.

\section{DSC Heating curves of VCO}

The overlay of DSC curves presented in Figure 1 compares the DSC heating curves of VCO (Curve-A) and PO (Curve-B). Based on the illustration, DSC heating curves of VCO and PO were significantly different; VCO displayed one major exothermic peak at $23.78{ }^{\circ} \mathrm{C}$ with a shoulder peak at $14.20{ }^{\circ} \mathrm{C}$. The existence of the shoulder peak close to the major endothermic peak is attributed to the co-melting of two different groups of TAG molecules. While the smaller shoulder peak indicates the lower melting TAG components of VCO, the major peak indicates the higher melting TAG components. The overlay of DSC curves presented in Figure 2 showed the gradual deviations in DSC features caused by adulteration. The changing proportion of adulterant affected all DSC parameters of the two peaks. As discussed before by previous researchers, the changing pattern of thermal profiles of the samples especially in peak 1 (major peak) and peak 2 (shoulder peak) could be attributed to the TAG compositional changes caused by adulteration. With the increasing percentage of $\mathrm{PO}$, the shoulder peak started to flatten forming a clear separation from the major peak. In Peak-1 (the major peak), there was remarkable shift in the peak temperature from 23.78 to $19.09^{\circ} \mathrm{C}$. The reduction of its peak area (A) from 426.8 to $298.4 \mathrm{~mJ}$, the decrease in the onset temperature $(\mathrm{O})$ from 16.75 to $14.25^{\circ} \mathrm{C}$ and the decline of the peak height $(\mathrm{H})$ from 7.16 to $4.4 \mathrm{~mW}$ caused a reduction in the sharpness of the peak (Table 1). In Peak-2 (the shoulder peak), there was remarkable shift in the peak temperature from 14.20 to $9.20^{\circ} \mathrm{C}$ while the peak area (A) has increased from 11.34 to $42.38 \mathrm{~mJ}$. Apart from these, the decreases in onset temperature (O) from 9.57 to $4.43^{\circ} \mathrm{C}$ and the peak height $(\mathrm{H})$ from 1.64 to $0.41 \mathrm{~mW}$ caused reduction in the sharpness of the peak.

The linearities between individual DSC parameter and the varying level of the adulterant are compared as shown in Table 1. Pearson correlation analysis showed that all four DSC parameters of peak-1 namely, $\mathrm{A}, \mathrm{T}, \mathrm{H}$, and $\mathrm{O}$ displayed good correlation. However, for peak-2, the only DSC parameter showing good correlation was $\mathrm{T}$ (Table 1). When each of the DSC parameter of peak-1 was subjected to stepwise multiple regression analysis for quantification purpose (Table 2), the best regression model obtained was $\mathrm{Y}=-0.161$ Temperature $+14.0\left[\mathrm{R}^{2}=0.993(\mathrm{p}<0.0001)\right.$ and $\mathrm{SE}=0.17]$. This predictive model not only give high correlation coefficient with good confidence limit but also yielded a low value for standard error (SE). According to Table 2, other regression models obtained using peak onset temperature, peak area and peak height were found to display weaker correlation.

\section{DSC Cooling curves of VCO}

The overlay of DSC curves presented in Figure 3 compares the DSC cooling curves of VCO (Curve-A) and PO (Curve-B). Based on the illustration, DSC heating curves of VCO and PO were significantly different; VCO displayed two overlapping exothermic peaks at $3.56^{\circ} \mathrm{C}$ and $-0.89^{\circ} \mathrm{C}$. The occurrence of two overlapping exothermic peaks was the direct result of cocrystallization of TAG molecules, which were closely similar in their melting temperatures. Based on the illustration in Figure 4, addition of PO to VCO caused the two overlapping peaks to undergo morphological changes in their thermal behavior. As a notable feature, no new peak correspond to the presence of adulterant was appeared. When percentage of PO increased in the admixture, there was a slight shift in the peak temperature of peak-1 (the major peak) from 3.56 to $1.95^{\circ} \mathrm{C}$ and increase in the peak area (A) from 112.47 to $190.23 \mathrm{~mJ}$. Simultaneouly, the onset temperature $(\mathrm{O})$ tended to decrease from 5.93 to $2.67^{\circ} \mathrm{C}$ while the peak height $(\mathrm{H})$ went up from 4.89 to $10.31 \mathrm{~mW}$ (Table 1). In Peak-2, there was a shift in peak temperature $(\mathrm{T})$ from - 


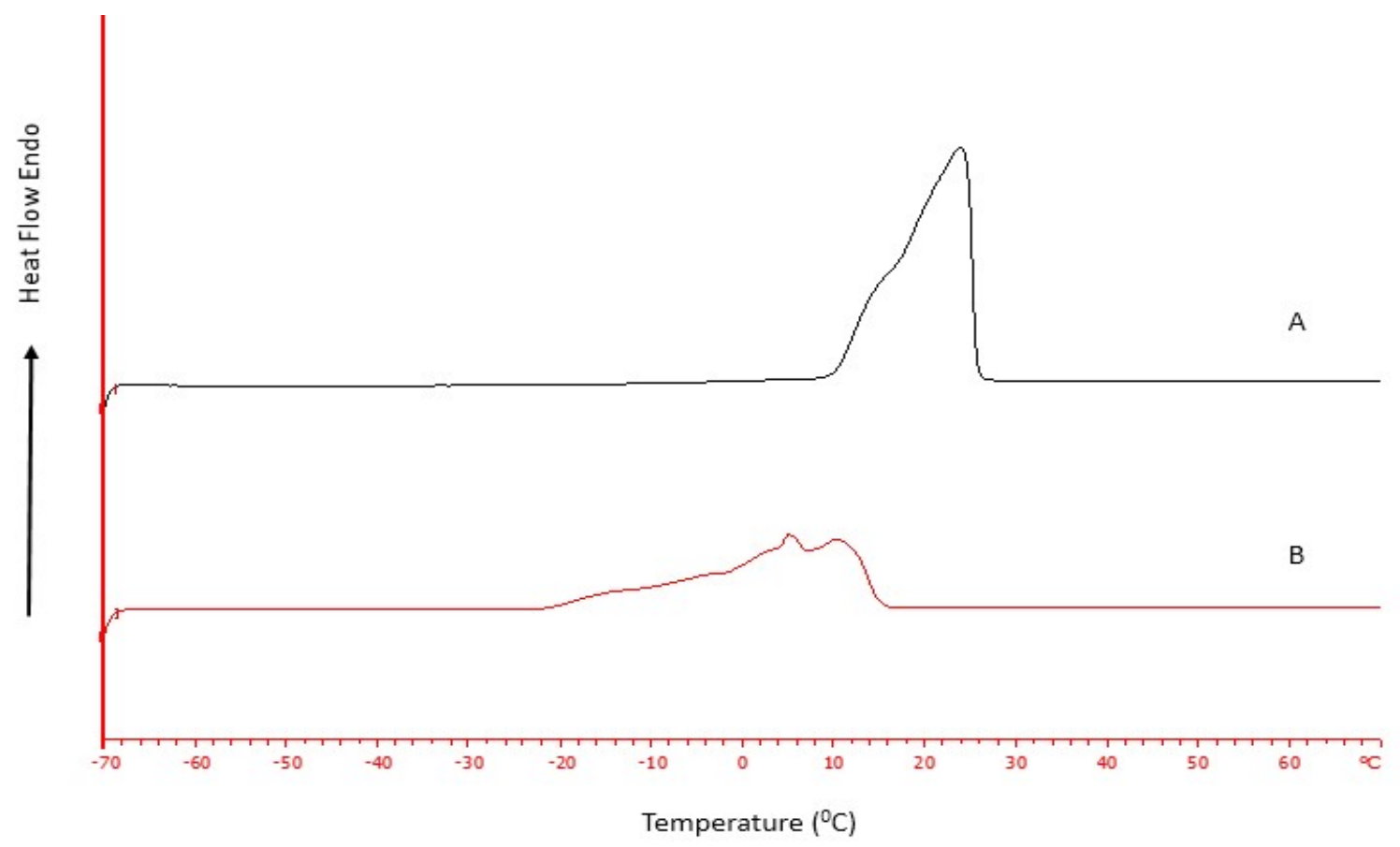

Figure 1. DSC heating curves of (A) virgin coconut oil (VCO) and (B) palm olein (PO)

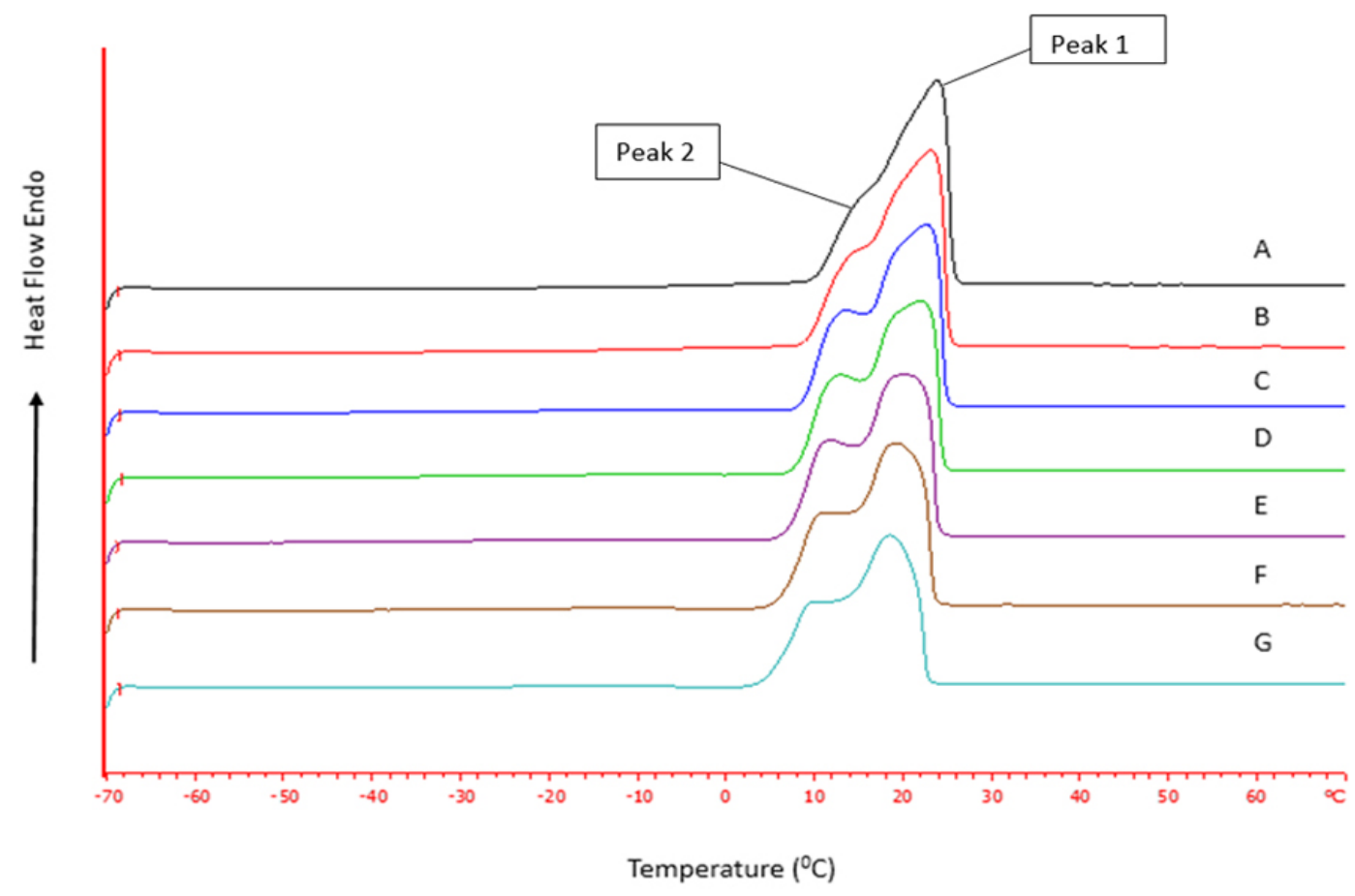

Figure 2. DSC heating curves of (A) virgin coconut oil (VCO), (B) VCO adulterated with 5\% palm olein (PO), (C) 10\% PO, (D) 15\% PO, (E) 20\% PO, (F) 25\% PO, and (G) $30 \%$ PO 
Cord 2019, 35 (1)

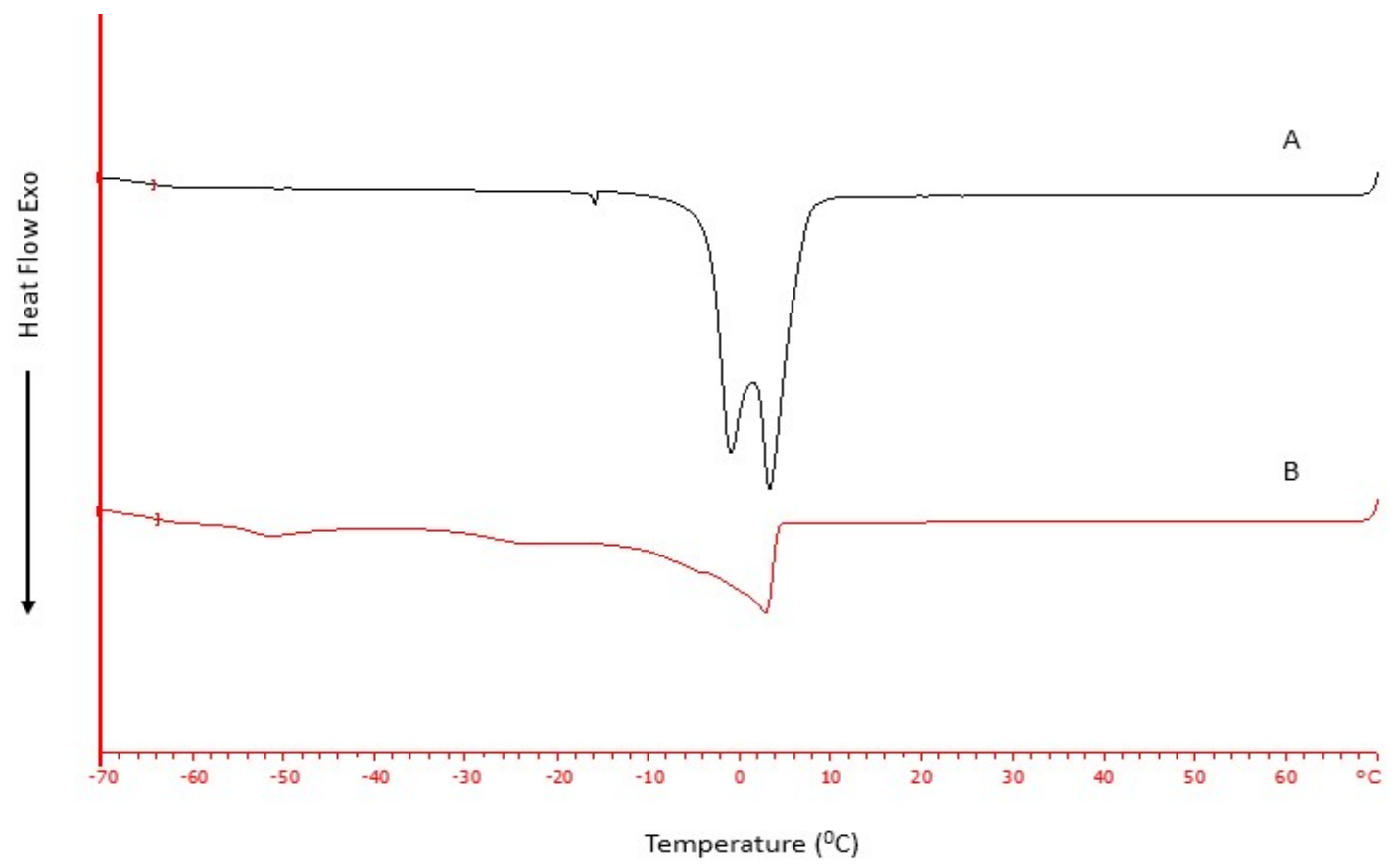

Figure 3. DSC cooling curves of (A) virgin coconut oil (VCO) and (B) palm olein (PO)

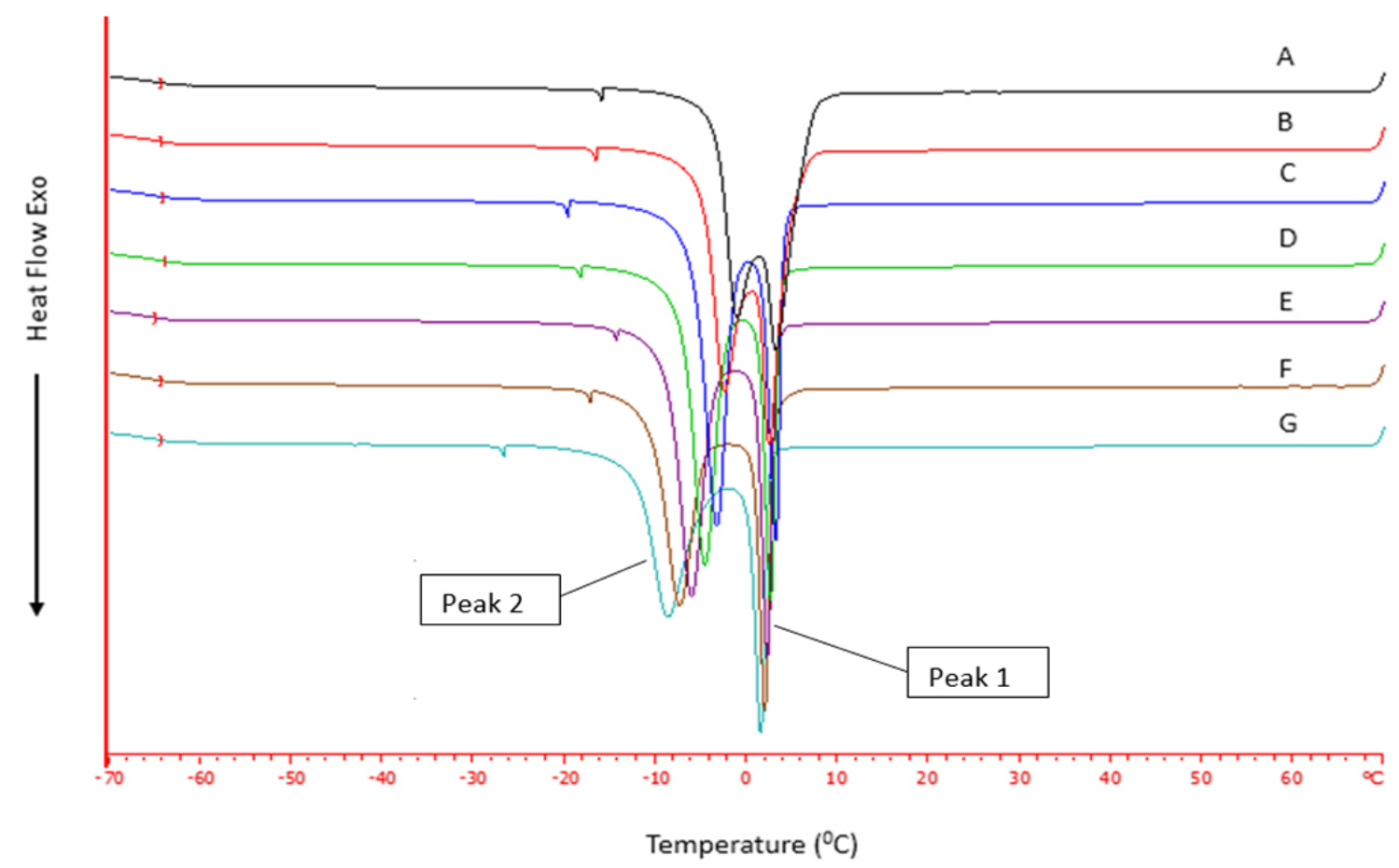

Figure 4. DSC cooling curves of (A) virgin coconut oil (VCO), (B) VCO adulterated with $5 \%$ palm olein (PO), (C) 10\% PO, (D) 15\% PO, (E) 20\% PO, (F) 25\% PO, and (G) $30 \%$ PO 
Cord 2019, 35 (1)

Table 1. Pearson correlation coefficient between \% level of adulterant and each of DSC paramameter associated with peaks in the cooling and heating curves ${ }^{1}$

\begin{tabular}{|c|c|c|c|c|}
\hline Curve Type & Peak & $\begin{array}{c}\text { DSC } \\
\text { Parameter }\end{array}$ & DSC Value Range & $\begin{array}{l}\text { Correlation } \\
\text { Coefficient }\end{array}$ \\
\hline & & $\mathrm{A}$ & 298.39 to 426.88 & $-0.947(\mathrm{p}<0.0001)$ \\
\hline \multirow[t]{8}{*}{ Heating } & Peak 1 & $\mathrm{~T}$ & 19.09 to 23.78 & $-0.977(\mathrm{p}<0.0001)$ \\
\hline & & $\mathrm{H}$ & 4.4 to 7.16 & $-0.996(\mathrm{p}<0.0001)$ \\
\hline & & $\mathrm{O}$ & 14.25 to 16.75 & $-0.979(\mathrm{p}<0.0001)$ \\
\hline & & A & 11.35 to 42.38 & $+0.505(\mathrm{p}<0.248)$ \\
\hline & Peak 2 & $\mathrm{~T}$ & 9.2 to 14.2 & $-0.996(\mathrm{p}<0.0001)$ \\
\hline & & $\mathrm{H}$ & 0.41 to 1.64 & $+0.524(\mathrm{p}<0.228)$ \\
\hline & & $\mathrm{O}$ & 4.43 to 9.57 & $-0.871(\mathrm{p}<0.011)$ \\
\hline & & A & -190.23 to -112.47 & $+0.337(\mathrm{p}<0.144)$ \\
\hline \multirow[t]{7}{*}{ Cooling } & Peak 1 & $\mathrm{~T}$ & 1.95 to 3.56 & $-0.898(\mathrm{p}<0.006)$ \\
\hline & & $\mathrm{H}$ & 4.89 to 10.31 & $+0.701(\mathrm{p}<0.079)$ \\
\hline & & $\mathrm{O}$ & 2.67 to 5.93 & $-0.976(\mathrm{p}<0.0001)$ \\
\hline & & A & -300.58 to -67.44 & $-0.644(\mathrm{p}<0.118)$ \\
\hline & Peak 2 & $\mathrm{~T}$ & -8.52 to -0.89 & $-0.999(\mathrm{p}<0.0001)$ \\
\hline & & $\mathrm{H}$ & 4.4 to 11.55 & $+0.217(\mathrm{p}<0.639)$ \\
\hline & & $\mathrm{O}$ & -5.23 to 0.99 & $-0.998(\mathrm{p}<0.0001)$ \\
\hline
\end{tabular}

${ }^{1}$ Abbreviation: A, peak area; $\mathrm{T}$, peak temperature; $\mathrm{H}$, peak height; $\mathrm{O}$, peak onset temperature 
Table 2. Summary of stepwise regression analysis carried for predictive model of PO content using DSC parameters of the heating curve ${ }^{1}$

\begin{tabular}{|c|c|c|c|}
\hline Model & Regression equation & $\mathrm{R}^{2}$ & SE \\
\hline & Peak 1 & & \\
\hline 1 & $\mathrm{Y}=-0.161$ Temperature +14.0 & $0.993(\mathrm{p}<0.0001)$ & 0.17 \\
\hline 2 & $\mathrm{Y}=0.905$ Area +33.9 & $0.255(\mathrm{p}<0.042)$ & 18.32 \\
\hline 3 & $\mathrm{Y}=0.021$ Height +0.828 & $0.274(p<0.030)$ & 0.40 \\
\hline \multirow[t]{2}{*}{4} & $Y=-0.151$ Onset +10.5 & $0.759(\mathrm{p}<0.0001)$ & 1.00 \\
\hline & Peak 2 & & \\
\hline 1 & $\mathrm{Y}=-0.153$ Temperature +24.1 & $0.995(\mathrm{p}<0.0001)$ & 0.39 \\
\hline 2 & $\mathrm{Y}=-3.80$ Area +4.36 & $0.896(\mathrm{p}<0.0001)$ & 15.32 \\
\hline 3 & $Y=-0.0952$ Height +7.22 & $0.992(\mathrm{p}<0.0001)$ & 0.10 \\
\hline 4 & $Y=-0.0847$ Onset +17.0 & $0.957(\mathrm{p}<0.0001)$ & 0.21 \\
\hline
\end{tabular}

${ }^{1}$ Abbreviation: Y, \% of adulterant; SE, standard error

Table 3. Summary of stepwise regression analysis carried for predictive model of PO content using DSC parameters of the cooling curve ${ }^{1}$

\begin{tabular}{|c|c|c|c|}
\hline Model & Regression equation & $\mathrm{R}^{2}$ & SE \\
\hline \multicolumn{4}{|c|}{ Peak 1} \\
\hline 1 & $\mathrm{Y}=-0.0485$ Temperature +3.58 & $0.806(\mathrm{p}<0.0001)$ & 0.28 \\
\hline 2 & $Y=-2.24$ Area -1.57 & $0.375(\mathrm{p}<0.001)$ & 34.17 \\
\hline 3 & $Y=0.191$ Height +7.23 & $0.491(\mathrm{p}<0.006)$ & 2.30 \\
\hline 4 & $Y=-0.102$ Onset +5.52 & $0.953(\mathrm{p}<0.0001)$ & 0.27 \\
\hline \multicolumn{4}{|c|}{ Peak 2} \\
\hline 1 & $Y=-0.253$ Temperature -0.771 & $0.997(\mathrm{p}<0.001)$ & 0.15 \\
\hline 2 & $Y=-7.97$ Area -152 & $0.415(\mathrm{p}<0.103)$ & 111.95 \\
\hline 3 & $Y=0.055$ Height +6.89 & $0.047(\mathrm{p}<0.019)$ & 2.95 \\
\hline 4 & $Y=-0.214$ Onset +0.951 & $0.995(\mathrm{p}<0.0001)$ & 0.17 \\
\hline
\end{tabular}

Abbreviation: Y, \% of adulterant; SE, standard error 
0.89 to -8.52 while the peak area (A) increased from -67.44 to $-300.58 \mathrm{~mJ}$. In the meantime, onset temperature $(\mathrm{O})$ of this peak tended to decrease in value from 0.99 to $-5.23^{\circ} \mathrm{C}$ while the peak height $(\mathrm{H})$ fell from 4.4 to $6.02 \mathrm{~mW}$ leading to the reduction in the sharpness of the peak (Table 1).

Pearson correlation analysis showed that among the DSC parameters of peak-1, peak onset temperature $(\mathrm{O})$ displayed good correlation [-0.976; $\mathrm{p}<0.0001]$ while in peak-2, both peak temperature T $[-0.999 ; \mathrm{p}<0.000]$ and peak onset $\mathrm{O}[-0.998 ; \mathrm{p}<0.0001]$ were found to display good correlations. When each of the DSC parameter of peak-1 was subjected to stepwise multiple regression analysis for quantification purpose (Table 3 ), $\mathrm{Y}=-0.102$ Onset $+5.52\left[\mathrm{R}^{2}\right.$ $=0.953(\mathrm{p}<0.0001)$ and $\mathrm{SE}=0.27]$ was obtained as the best regression model. This predictive model not only give high correlation coefficient with good confidence limit but also a very low $\mathrm{SE}$ value. When the same procedure was repeated for DSC parameters associated with peak-2, the following two regression models having high correlation coefficient with good confidence limit and very low SE values were obtained:

$$
\begin{aligned}
\mathrm{Y}= & -0.214 \text { Onset }+0.951\left[\mathrm{R}^{2}=0.995(\mathrm{p}<\right. \\
& 0.0001) \text { and } \mathrm{SE}=0.17] \\
\mathrm{Y}= & -0.253 \text { Temperature }-0.771\left[\mathrm{R}^{2}=0.997\right. \\
& (\mathrm{p}<0.001) \text { and } \mathrm{SE}=0.15]
\end{aligned}
$$

According to Table 3, other regression models obtained using peak area and peak heights were found to display weaker correlation.

\section{Conclusions}

This study investigated the use of DSC thermal analysis for detecting adulteration in VCO by PO. Comparative DSC curves of both heating and cooling processes of $\mathrm{VCO}$ and $\mathrm{PO}$ were significantly different. Owing to this reason, DSC curves of VCO were found to deviate remarkably after adulteration with PO. It is believed that the changing proportion of saturated to unsaturated TAG molecular ratio caused these significant changes in thermal transitions and the associated DSC parameters of
VCO. According to Pearson correlational analysis, DSC parameters namely peak temperature, onset, and peak height were sensitive parameters based on the heating curves while peak temperature, onset, and peak height was the most sensitive parameters based on the cooling curves. For quantitative estimation of adulteration levels, predictive models based on DSC parameters namely onset and peak temperature associated with cooling curve were found to have high correlation coefficient with lower standard error values.

\section{Acknowledgments}

Authors gratefully acknowledge financial support and research facilities given by Universiti Putra Malaysia.

\section{References}

Balawan, D.D. and Chapman, K.R. 2006. Virgin Coconut Oil Production Manual for Micro and Village Scale Processing, FAO Regional Office Thailand.

Dyszel, S.M., and Baish, S.K. 1992. Characterization of tropical oils by DSC. Thermochim. Acta 212: 41-49

Jayasundera, M., Yalegama, C., Dharmasena, A. and Jayathilake, S. 2003. Dry processing of virgin coconut oil. In: Report of the Coconut Research Institute of Sri Lanka for 2003, Jayasekera, C. and Jayamanne, J.M.T.D. (Eds.) pp. 168-169, Lunuwila.

Marina, A.M. Che Man, Y.B. Nazimah, S.A.H., and Amin, I. 2009. Monitoring the adulteration of virgin coconut oil by selected vegetable oils using differential scanning calorimetry. Journal of Food Lipids 16: 50-61.

Manaf, M.A., Che Man, Y.B., Hamid, N.S.A., Ismail, A. and Abidin, S.Z. 2007. Analysis of adulteration of virgin coconut oil by palm kernel olein using Fourier transform infrared spectroscopy. Journal of Food Lipids 14: 111-121.

Marikkar, J.M.N. 2015. DSC as a valuable tool for the evaluation of oils and fats adulterations. In: Differential Scanning 
Calorimetry: Applications in Fat and Oil Technology, Emma Chiavaro (Editor). CRC Press, Taylor \& Francis Group, Florida, USA. pp. 159-178. ISBN: 9781466591523

Marikkar, J.M.N., Saraf, D. and Dzulkifly, M.H. 2013. Effect of fractional crystallization on composition and thermal behavior of coconut oil. International Journal of Food Properties 16: 1284-1292.

Nevin, K.G. and Rajamohan, T. 2004. Beneficial effects of virgin coconut oil on lipid parameters and in vitro LDL oxidation. Clin. Biochem. 37, 830-835.
Nagao, K. and Yanagita, T. (2010). Mediumchain fatty acids: Functional lipids for the prevention and treatment of the metabolic syndrome. Pharmacological Research. 61 (3): 208-212.

SAS Statistical Analysis System User's Guide: Basic Statistics 1998. SAS Institute Inc. Cary, NC.

Xu, B., Li, P., Ma, F., Wang, X., Matthäus, B., Chen, R. and Zhang, Q. 2015. Detection of virgin coconut oil adulteration with animal fats using quantitative cholesterol by $\mathrm{GC} \times \mathrm{GC}-\mathrm{TOF} / \mathrm{MS}$ analysis. Food Chemistry 178:128-35. 